\title{
Algorithmic complexity as an index of cortical function in awake and pentobarbital-anesthetized rats
}

\author{
Fu-Zen Shaw a, Ruei-Feng Chen ${ }^{\mathrm{b}}$, Hen-Wai Tsao ${ }^{\mathrm{c}}$, Chen-Tung Yen ${ }^{\mathrm{b}, *}$

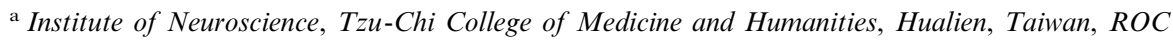 \\ ${ }^{\mathrm{b}}$ Department of Zoology, National Taiwan University, Taipei, Taiwan, ROC \\ ${ }^{c}$ Department of Electrical Engineering, National Taiwan University, Taipei, Taiwan, ROC
}

Received 14 December 1998; received in revised form 20 July 1999; accepted 13 August 1999

\begin{abstract}
This study introduces algorithmic complexity to measure characteristics of brain functions. The EEG of the rat was recorded with implanted electrodes. The normalized complexity value was relatively independent of data length, and it showed a simpler and easier calculation characteristic than other non-linear indexes. The complexity index revealed significant differences among awake, asleep, and anesthetized states. It may be useful in tracking short-term and long-term changes in brain functions, such as anesthetized depth, drug effects, or sleep-wakefulness. (C) 1999 Elsevier Science B.V. All rights reserved.
\end{abstract}

Keywords: Chaotic; Chronic recording; Consciousness; Nonlinear; Sleep

\section{Introduction}

The electroencephalogram (EEG) reflects the activity of millions of neurons, in particular, radially oriented pyramidal neurons of the cortex. From this viewpoint, the EEG does contain information regarding phenomena at the network level, for example, the degree of synchronization within local networks or the coupling between distant networks. Many derivations (for example, statistical moments and spectral distribution in various special bandwidths) have been used to estimate the characteristics of the EEG, and to relate these indexes to different functional states of the brain. In the last decade, non-linear analysis has provided new insights in neural mechanisms underlying cortical network functions (Basar, 1990; Elbert et al., 1994). Several methods, such as correlation dimension (Grassberger and Procaccia, 1983; Theiler and Rapp, 1996), Lyapunov exponent (Roschke et al., 1993), and complexity/entropy (Kaspar and Schuster, 1987; Destexhe, 1990; Paulus et al., 1990; Rapp et al., 1994; Xu and $\mathrm{Wu}, 1994)$, are used to evaluate different mental condi-

\footnotetext{
* Corresponding author. Tel.: + 886-2-23630231 ext. 3322; fax: $+886-2-23636837$.

E-mail address: ctyen@ccms.ntu.edu.tw (C.-T. Yen)
}

tions from the unit firing pattern, EEG, and behavior. However, estimations of correlation dimension and Lyapunov exponent are extremely sensitive to noise in the data. They can only be reliably applied when large, noise-free data sets are available (Eckmann and Ruelle, 1992; Theiler and Rapp, 1996). Moreover, calculations of these indexes are relatively complicated. A simple calculation of the algorithmic complexity is introduced herein. The complexity of the EEG is calculated in different functional states.

\section{Algorithm of complexity calculation}

Several alternative definitions of complexity have been published (Kolmogorov, 1965; Chaitin, 1974), for example, context-free grammar. According to Kolmogorov's definition, the complexity of a given string of zeros and ones is given by the number of bits of the shortest computer program which can generate this string. In other words, the complexity of a message can be quantified by determining a measure of the length of a program required to generate the message. We used the complexity measure, $c(n)$, of Lempel and Ziv (1976), which is an appropriate measure of the Kolmogorov complexity. Lempel and Ziv (1976) found that 
only two operations, i.e. copy and insert, are necessary to generate the message of all possible programs. The calculation of $c(n)$ proceeds as follows. Let us assume that a given string $\mathrm{s}_{1} \mathrm{~s}_{2} \ldots . \mathrm{s}_{\mathrm{n}}{ }^{\bullet}$ has been reconstructed by the program up to the digit $\mathrm{s}_{\mathrm{k}}$. The string up to $\mathrm{s}_{\mathrm{k}}$ is denoted by $S=s_{1} s_{2} . . s_{k}$, where the dot indicates that $s_{k}$ is newly inserted. In order to check that the rest of the string, i.e. $s_{k+1} \ldots s_{n}$, could be reconstructed by simple copying from S, Lempel and Ziv (1976) proceeded with the following steps. First, one takes $\mathrm{IN} \equiv \mathrm{s}_{\mathrm{k}+1}$ and asks whether this term is contained in the vocabulary (i.e. in one of the substrings or words) of the string S. If IN is contained in the vocabulary of $\mathrm{S}$, it can simply be obtained by copying a word of S. That is equivalent to the question of whether IN is contained in the vocabulary $\mathrm{v}\left(\mathrm{SIN}^{\prime}\right)$ of $\mathrm{SIN}^{\prime}$, where $\mathrm{SIN}^{\prime}$ denotes the string which is composed of S and IN and the substring which the last digit has been deleted (i.e. $\mathrm{SIN}^{\prime}=\mathrm{S}$ here). This formulation of the question can be generalized to situations where IN contains two (i.e. $\mathrm{s}_{\mathrm{k}+1} \mathrm{~s}_{\mathrm{k}+2}$ ) or more elements. For example, if $\mathrm{s}_{\mathrm{k}+1}$ can be copied from the vocabulary of $\mathrm{S}$. Then, we next ask whether $\mathrm{IN}=$ $\mathrm{S}_{\mathrm{k}+1} \mathrm{~S}_{\mathrm{k}+2}$ is contained in the vocabulary of $\mathrm{SIN}^{\prime}$ and so on until IN becomes so large that it can no longer be obtained by copying a word from $\mathrm{v}\left(\mathrm{SIN}^{\prime}\right)$, and one has to insert a new digit. The number $\mathrm{c}$ of production steps to create a string is used as a measure of the complexity of a given string.

The procedure is best described by considering some specific examples. If we have a sequence which contains only zeros, we could intuitively say that it should have the smallest possible complexity of all strings. One has only to insert the first zero and then one can reconstruct the whole string by copying this digit, i.e.

1. initial setting $S=0 \rightarrow 0 \bullet$

2. $\mathrm{S}=0, \mathrm{IN}=0, \mathrm{SIN}=00, \mathrm{SIN}^{\prime}=0, \mathrm{IN} \in \mathrm{v}\left\{\mathrm{SIN}^{\prime}\right\} \rightarrow$ $0 \cdot 0$

3. $\mathrm{S}=0, \quad \mathrm{IN}=00, \quad \mathrm{SIN}=000, \quad \mathrm{SIN}^{\prime}=00$, $\mathrm{IN} \in \mathrm{V}\left\{\mathrm{SIN}^{\prime}\right\} \rightarrow 00 \bullet 00$

4. $\mathrm{S}=0, \quad \mathrm{IN}=000, \quad \mathrm{SIN}=0000, \quad \mathrm{SIN}^{\prime}=000$, $\mathrm{IN} \in \mathrm{V}\left\{\mathrm{SIN}^{\prime}\right\} \rightarrow 0 \bullet 000$

The complexity $(c)$ is equal to the number of parts of the string that are separated by dots. The complexity of this string is $c=2$ here.

Similarly, the complexity of a sequence 0010110 can be determined via the following steps.

1. initial setting $S=0 \rightarrow 0$ •

2. $\mathrm{S}=0, \mathrm{IN}=0, \mathrm{SIN}=00, \mathrm{SIN}^{\prime}=0, \quad \mathrm{IN} \in \mathrm{v}\left\{\mathrm{SIN}^{\prime}\right\} \rightarrow$ $0 \bullet 0$

3. $\mathrm{S}=0, \quad \mathrm{IN}=01, \quad \mathrm{SIN}=001, \quad \mathrm{SIN}^{\prime}=00$, $\mathrm{IN} \notin \mathrm{v}\left\{\mathrm{SIN}^{\prime}\right\} \rightarrow 0 \bullet 01 \cdot$

4. $\mathrm{S}=001, \quad \mathrm{IN}=0, \quad \mathrm{SIN}=0010, \quad \mathrm{SIN}^{\prime}=001$, $\mathrm{IN} \in \mathrm{V}\left\{\mathrm{SIN}^{\prime}\right\} \rightarrow 0 \bullet 01 \cdot 0$

5. $\mathrm{S}=001, \quad \mathrm{IN}=01, \quad \mathrm{SIN}=00101, \quad \mathrm{SIN}^{\prime}=0010$, $\mathrm{IN} \in \mathrm{V}\left\{\mathrm{SIN}^{\prime}\right\} \rightarrow 0 \bullet 01 \bullet 01$
6. $\mathrm{S}=001, \quad \mathrm{IN}=011, \quad \mathrm{SIN}=001011, \quad \mathrm{SIN}^{\prime}=00101$, $\mathrm{IN} \notin \mathrm{v}\left\{\mathrm{SIN}^{\prime}\right\} \rightarrow 0 \bullet 01 \bullet 011$

7. $\mathrm{S}=001011, \mathrm{IN}=0, \mathrm{SIN}=0010110, \mathrm{SIN}^{\prime}=001011$, $\mathrm{IN} \in \mathrm{V}\left\{\mathrm{SIN}^{\prime}\right\} \rightarrow 0 \bullet 01 \cdot 011 \bullet 0$

The complexity value of the string 0010110 is $c=4$.

We have introduced the example of the two-stage sequence (binary string). In real situations, two-stage classification is not suitable for all kinds of responses, such as, the sleep stage and the behavior responses to nociceptive stimuli. A nonbinary example will be considered below. The same procedures can be used for nonbinary strings. The nonbinary string becomes

$11241143211443224 \rightarrow 1 \cdot 12 \cdot 4 \cdot 114 \cdot 3 \cdot 2 \cdot 1144 \cdot 322 \cdot 4$

The nonbinary string can be separated into 9 parts by dots. The complexity of the string is $c=9$. For the computation of random sequence of length $n$, the computer time is on the order of $n^{2}$. For a string only consisting of zero, the computer time is on the order of $n$. Therefore, the computer time for a general information-limited sequence will be of an order between $n$ and $n^{2}$.

Since the number of bits of the shortest string is obtainable, the complexity value is dependent on the length of the string. In order to obtain a quantitative and robust measure, the complexity must be constrained (normalized) in the range of $[0,1]$. In theoretical considerations, almost all random strings have a maximal complexity. Lempel and Ziv (1976) have shown that the complexity $c(n)$ of random strings converges to the same value:

$\operatorname{Lim}_{n \rightarrow \infty} c(n)=b(n) \cong \frac{n}{\log _{2} n}$

where $b(n)$ shows the asymptotic behavior of $c(n)$ for a random string. We can normalize $c(n)$ via this limit. In other words, we will only consider the finite ratio $0 \leq c(n) / b(n) \leq 1$, instead of $c(n)$.

\section{Surgery, recording, and data analysis procedures}

Male Wistar rats (250 to $300 \mathrm{~g} ; n=4$ ) were anesthetized with sodium pentobarbital $\left(50 \mathrm{mg} \mathrm{kg}^{-1}\right.$, i.p.) initially. Ketamine was administered as necessary to maintain anesthesia. Once anesthesia was induced, the dorsal surface of the animal's head was shaved and the rat was placed in a stereotaxic apparatus. Connective tissue was scraped away from the skull surface. The skull was roughened with a bone curette and then cleaned with saline. After exposure of the skull, a small concavity on each marked position of the skull was drilled first and a stainless steel screw was securely driven into this position. Fourteen small screw electrodes ( $1 \mathrm{~mm}$ in diameter) were symmetrically arranged on both sides of the head separated by $3 \mathrm{~mm}$ inter-elec- 
trode distance. Special care was taken not to penetrate the dura. Monopolar recordings were made from these screws. A reference electrode was implanted $2 \mathrm{~mm}$ caudal to lambda. Dental cement was then applied in order to secure the connector in place and fasten it to the surface of the skull. Animals were injected with antibiotics (Combion-S, $60000 \mathrm{U} \mathrm{kg}^{-1}$, i.p.). The rats were then housed individually in cages.

One month following recovery from surgery, animals were placed in an acrylic recording chamber with a slit in the top. This chamber was designed to allow recording from unrestrained animals. The behavior of the rat could be clearly observed. We used a custom-fabricated multistrand printer cable line to connect the connector modules to the scalp of the rat and to amplifiers $(0.3-70 \mathrm{~Hz}$, gain $=2000)$ through a slit in the top cover of the chamber. To habituate the rat to the experimental chamber, the rat was put into the recording chamber five times ( $2 \mathrm{~h}$ per day) before each experiment. The animal was placed in the recording chamber for $30 \mathrm{~min}$ prior to the recording session. All surgical and recording procedures complied with NIH (USA) recommended procedures for animal use and care.

Rat EEG was digitized with a data acquisition card (AT-16-MIO-F5, National Instruments, Austin, TX). The sampling frequency was $250 \mathrm{~Hz}$. The data were stored on a computer hard disk and processed off-line. Data were partitioned into 8-s segments (2000 data points) with $50 \%$ overlapping to calculate the algorithmic complexity or statistical moments (see next section for the reason for choosing a 2000-data-point segment). EEG segments with movement artifacts or large potential drift were discarded. Their values were left blank in the post-processing procedure (statistical analysis). The first zero crossing point of the autocorrelation of the

Table 1

Comparisons of the complexity values calculated from randomly generated and realistic data sequences partitioned into binary sequences about the threshold value of median, mean, and midpoint, respectively ${ }^{\mathrm{a}}$

\begin{tabular}{llll}
\hline $\begin{array}{l}\text { Distribution/ } \\
\text { Signal }\end{array}$ & Median & Mean & Midpoint \\
\hline Uniform & $1.048 \pm 0.011^{\mathrm{a}}$ & $1.049 \pm 0.012$ & $1.052 \pm 0.013$ \\
Gaussian & $1.045 \pm 0.013$ & $1.048 \pm 0.013$ & $1.031 \pm 0.023$ \\
Exponential & $0.967 \pm 0.02$ & $0.914 \pm 0.053$ & $0.398 \pm 0.14$ \\
Poisson & $1.039 \pm 0.011$ & $1.029 \pm 0.018$ & $0.733 \pm 0.112$ \\
Random phase & $1.049 \pm 0.011$ & $1.049 \pm 0.011$ & $1.024 \pm 0.043$ \\
3-Periodic & $0.345 \pm 0.015$ & $0.343 \pm 0.014$ & $0.348 \pm 0.014$ \\
$\quad$ wave & & & \\
Wakeful EEG & $0.602 \pm 0.035$ & $0.600 \pm 0.036$ & $0.560 \pm 0.093$ \\
Wakeful EEG & $0.604 \pm 0.035$ & $0.599 \pm 0.038$ & $0.565 \pm 0.07$ \\
\hline
\end{tabular}

\footnotetext{
${ }^{\text {a }}$ Values are means \pm SD from 30 sets of data sequences.

b 3-periodic wave: $\sin (8+\theta 1)+\sin (8 \sqrt{ } 2+\theta 2)+\sin (8 \sqrt{ } 3+\theta 3)$; $\theta 1, \theta 2$ and $\theta 3$ are random initial phases.

${ }^{\mathrm{c}}$ Wakeful EEG with phase randomizing surrogates.
}

selected data segment was used as a time lag to plot the phase portrait (Basar, 1990; Elbert et al., 1994). A moving average was used to smooth the complexity sequence in awake-pentobarbital-treated process. Detailed descriptions of the analytic program were described in a previous investigation (Shaw et al., 1999). All programs were developed with Labview (National Instruments).

\section{Properties of algorithmic complexity}

Before calculating the complexity of the data, we had to transfer the data sequence into a symbol sequence. If data transformation is inappropriate, spurious results will be obtained. Eight types of signals were used to evaluate the most suitable reference point for the partitioning of the data sequence to symbol sequence. The computational results depicted in Table 1 were obtained after these signals were reduced to a binary sequence by partitioning about the median, the mean, or the midpoint. For instance, if the data value was less than the mean, it was assigned the symbol ' 0 '. On the other hand, if the data value was greater than or equal to the mean, it was assigned the symbol ' 1 '. Partitions about the median and the midpoint followed the same procedure. With randomly generated signals, the best partition method should reveal the most randomness of the original data and the least variation. Partitioning about the median met these criteria the best (Table 1, rows $1-5)$. Partitioning asymmetrically distributed data about the midpoint showed a misleading indication of structure (e.g. exponential distribution in Table 1). In addition, the complexity values of the 3-periodic wave and wakeful EEG with/without phase randomized surrogates showed consistent results for the three different partitioning methods (Table 1, rows 6-8). Therefore, all binary symbol sequences reported in this article were constructed by partitioning about the median.

The stability of the calculated complexity value was also evaluated. Twenty data sets composed of Gaussian random noises were used to evaluate the characteristics of the complexity calculations under different information contents (different lowpass filter bands) and different data lengths. Obviously, the complexity value decreased as the information content of the data sequence decreased (i.e. with the decrease of the cut-off frequency) (Fig. 1A). And the complexity value decreased exponentially with an increase of the size of the data set (Fig. 1A). The normalized complexity would be overestimated for a shorter data segment. The complexity value reached a plateau about a data length of 2000 . For the 3-periodic wave and wakeful EEG, the complexity values also showed an exponential decay relationship as the size of the data lengths increased (Fig. 1B). On average, the complexity value approaches a 


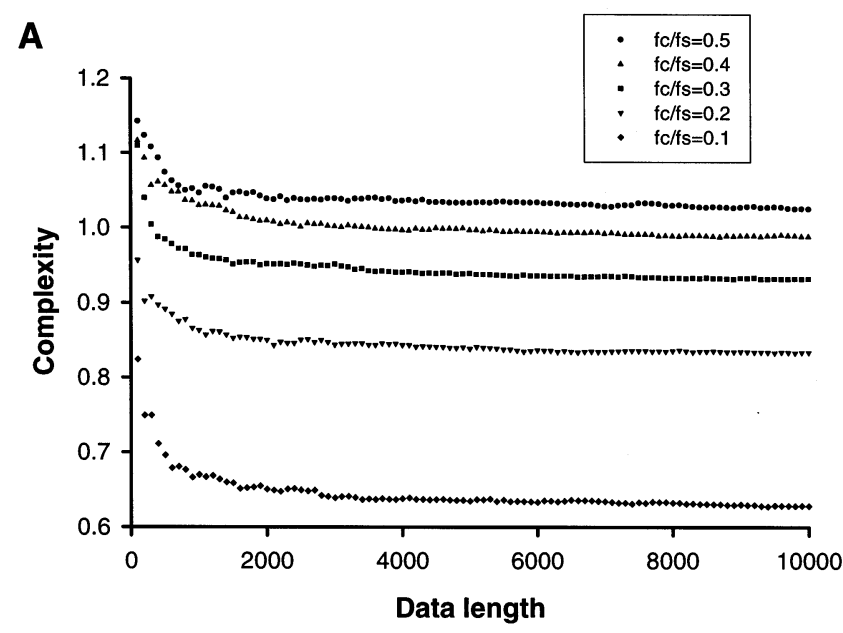

B

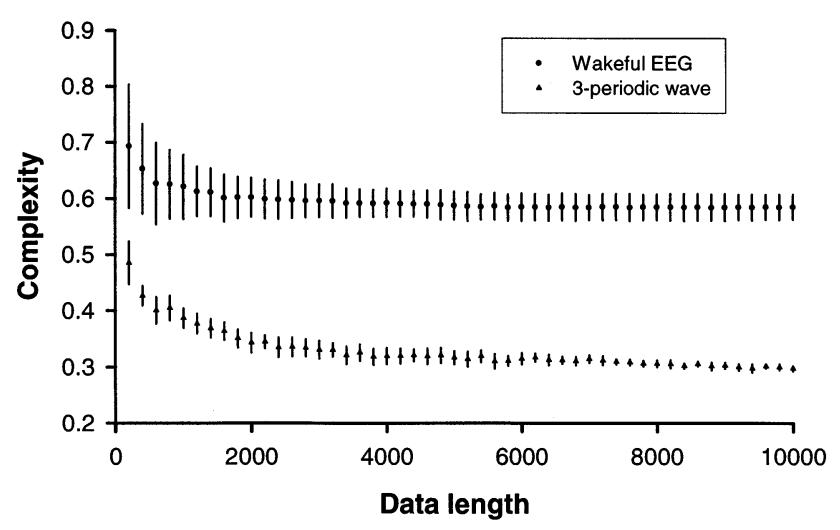

Fig. 1. Complexities of data segments of different lengths. (A) Complexities of data segments of different lengths under five information contents. Gaussian white noises with five different cut-off frequencies were used. Note that the complexity value approaches the plateau of the relation curve as data points near 2000 under all five conditions; fc, cut-off frequency; fs, sampling frequency. (B) Complexities of 3 -periodic wave and EEG in different data lengths. The complexity value (mean $\pm \mathrm{SD}$ ) shows convergence as it approaches 2000 data points. The sampling frequency is $250 \mathrm{~Hz}$. The 3-periodic wave $=$ $\sin (8+\theta 1)+\sin (8 \sqrt{2}+\theta 2)+\sin (8 \sqrt{ } 3+\theta 3) ; \quad \theta 1, \theta 2$ and $\theta 3$ are random initial phases.

plateau about a data length of 2000. On the other hand, the EEG with long data length has a risk to remain stationary. Accordingly, in the following part of the present study, we used a data size of 2000 (i.e. 8-s segment).

\section{Complexities of different functional states}

To evaluate the usefulness of the algorithmic complexity value, the temporal sequence and the phase diagram of both the simulated waveform (pure sine wave) and the EEG in conscious, sleep, and anesthetized conditions were analyzed (Fig. 2). The phase diagram of the sinusoidal waveform is a limited cycle
(Fig. 2A). The complexity of the sinusoidal waveform was $0 \pm 0.038$ (mean $\pm \mathrm{SD}$; segment numbers $=30$ ). The prediction could be made precisely for the simple phase orbit (Basar, 1990; Elbert et al., 1994). Desynchronous low amplitude EEG was found in wakeful and alert condition, and its phase diagram shows dense and chaotic structure (Fig. 2B). The complexity value of the wakeful EEG was $0.595 \pm 0.025$ (segment numbers $=20$ ). Two synchronous high-amplitude oscillating cortical activities (sleep spindle and barbiturate oscillation) were selected to compare their complexity values. The sleep spindle is a waxing and waning EEG oscillating at $7-14 \mathrm{~Hz}$ for 1 to $3 \mathrm{~s}$ (Steriade et al., 1990). A regular and smooth orbit of the phase diagram is found for the sleep spindle cortical rhythm (Fig. 2C). The complexity value of the sleep spindle was $0.457 \pm 0.034$ (segment numbers $=10$ ). The barbiturate segment was selected about $30 \mathrm{~min}$ after intraperitoneal pentobarbital administration $\left(50 \mathrm{mg} \mathrm{kg}^{-1}\right)$. A clear perpendicular orbit is found on the phase diagram of the barbiturate oscillation rhythm (Fig. 2D). The phase orbit was more regular and repetitive than the sleep spindle. The complexity value of the barbiturate spindle was $0.367 \pm$ 0.012 (segment numbers $=15$ ). Although the cortical activity was oscillating at a similar frequency bandwidth, the complexity values of the sleep spindle and the barbiturate oscillation were significantly different (Student's $t$-test, $P<0.05$ ). From the above analysis, it can be seen that the complexity measure is able to differentiate these two different brain states, and that the underlying meaning of the algorithmic complexity is not completely determined by the frequency distribution of the data sequence (Rapp et al., 1994). Comparing wakeful and sleep EEGs (Fig. 2B, C), we found the complexity of wakeful cortical activity to be significantly higher than that of the sleep EEG (Student's $t$-test, $P<0.01)$. Furthermore, the complexity values of wakeful, sleep, and anesthetized EEG fell in the range of $0-1$. These results (shown in Fig. 2 and Table 1) imply that cortical activity is a chaotic signal, not a totally deterministic $(c \approx 0)$ or random $(c \approx 1)$ signal. The same result has been shown in the dimensional analysis (Basar, 1990; Elbert et al., 1994).

We also used the complexity value to track the dynamic change of the EEG after intraperitoneal pentobarbital administration $\left(50 \mathrm{mg} \mathrm{kg}^{-1}\right)$. The complexity values revealed intricate fluctuations during the 3-h recording period (Fig. 3). Generally, the complexity values decreased progressively as the rat lost consciousness $(10-80 \mathrm{~min})$, went into the minimum at the quasianesthesia state $(80-120 \mathrm{~min})$, then gradually returned to the control level as the rat showed wakeful behavior $(120-180 \mathrm{~min})$ (dashed line in Fig. 3). In the quasianesthesia state, the rat was unable to completely control its movements. During the recording period, the EEG showed a distinctive change in patterns (illus- 
trated in the upper part of Fig. 3). Desynchronous EEG was recorded at the beginning (1 min after injection), followed by a distinct high-voltage barbiturate spike wave (15 $\mathrm{min}$ after). The high-voltage barbiturate spikes were mostly negative sharp peaks (unipolar waves). In the quasi-anesthetic state, the EEG showed dense oscillations with unipolar negative spikes $(90 \mathrm{~min}$ after). The cortical potential became a high-amplitude bipolar wave (170 min after) when the animal had recovered and was able to move about the cage. Thus,
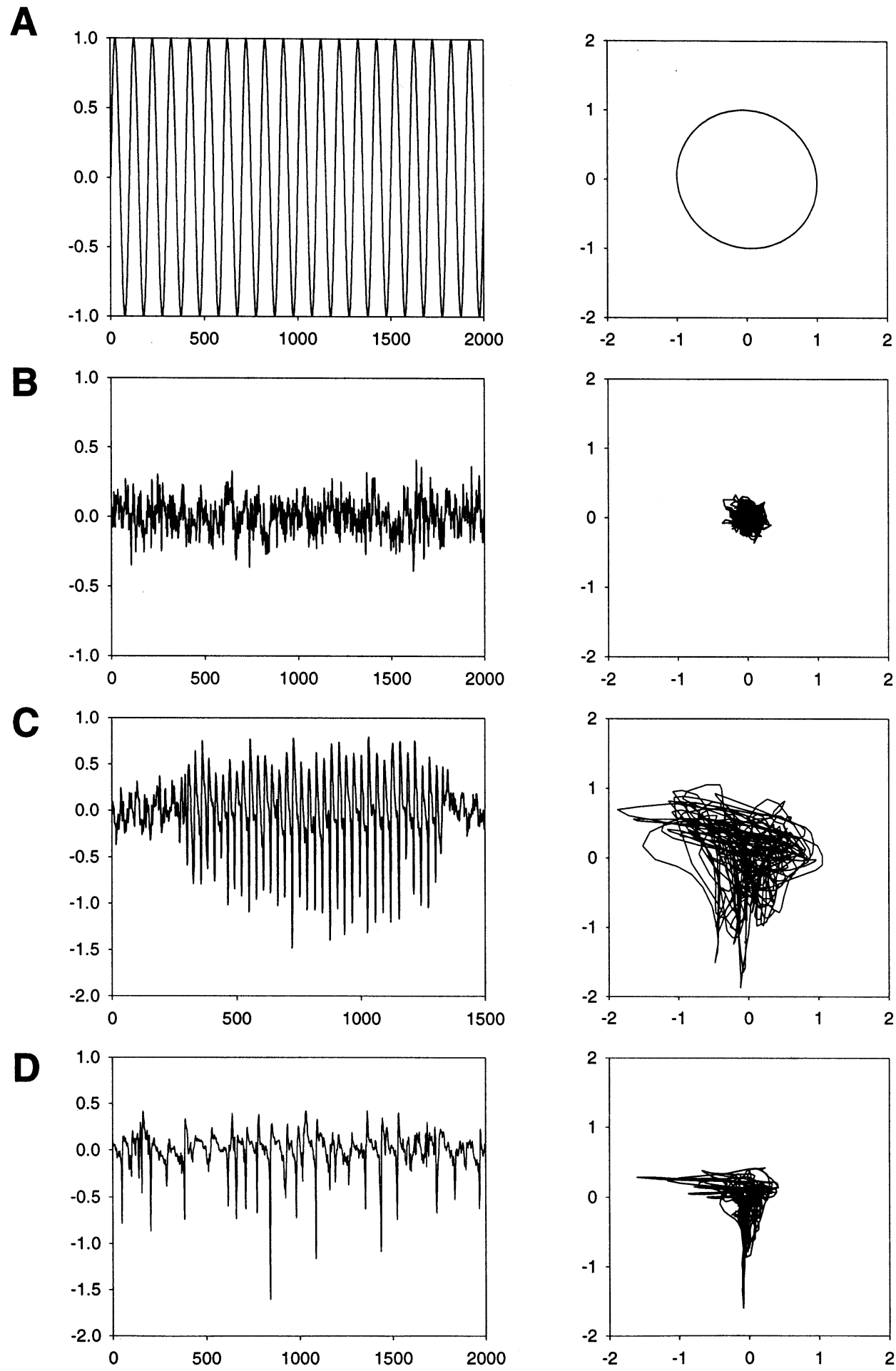

Fig. 2. Temporal patterns (left) and their phase plots (right) of four signals. These were: (A) simulated sinusoidal waveform; (B) EEG during wakefulness; (C) EEG sleep spindle; and (D) barbiturate spike activity. The complexity values are $0.022,0.607,0.483$, and 0.373 , respectively. The amplification of the EEG was 2000 for B, C, and D. 

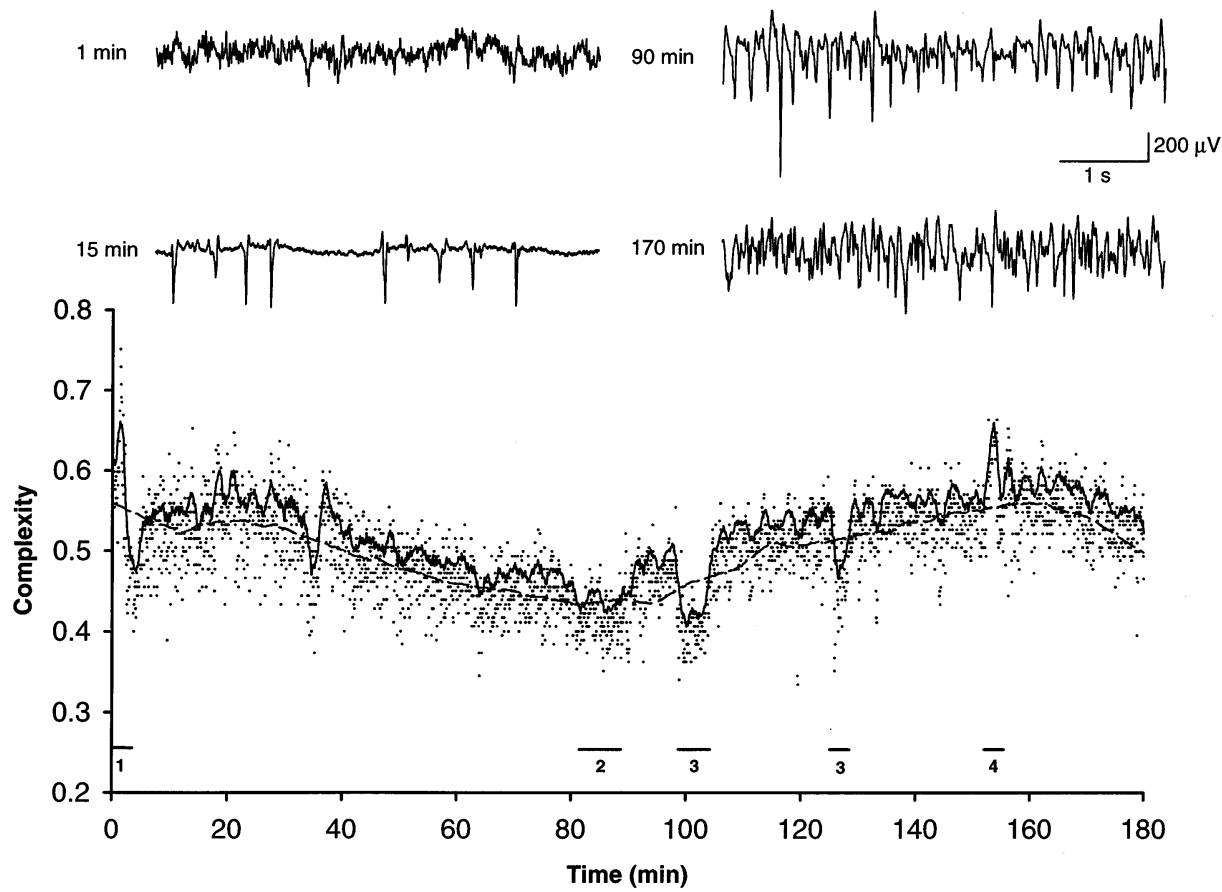

Fig. 3. Complexity values of EEG segments during a 3-h recording period after pentobarbital administration $\left(50 \mathrm{mg} \mathrm{kg}^{-1}\right.$, i.p. at time 0$)$. The complexity values (black dots) were calculated from 2000 selected data segments with 50\% overlap. Ten-point (solid line) and 300-point (dashed line) moving averages were used to smooth the original complexity trial. The complexity values show a biphasic change (increasing at first then dropping at the beginning phase) of wakefulness to anesthesia. The values decreased slowly in the anesthesia period (10-80 min), then increased from quasi-anesthesia $(80-120 \mathrm{~min})$ to the recovery period $(120-150 \mathrm{~min})$. Four segments of cortical activities were selected and expanded on the upper panel. Horizontal bars mark different behaviors: 1, the stage from wakefulness to anesthesia; 2 , movements of the head and tongue; 3 , body movements and crawling; 4, grooming and chewing.

there is general agreement of the complexity value versus the behavior of the rat.

For comparison purposes, the same EEG data set shown in Fig. 3 has been subjected to an analysis of traditional amplitude values. Four different statistical moments, namely, mean, variance, skewness, and kurtosis, were calculated. The comparison is shown in Fig. 4. Except mean values which remained close to zero throughout the anesthesia-recovering process, all other parameters showed dynamic changes. After injection of pentobarbital bolus, the values of skewness and kurtosis showed large changes which peaked at $10 \mathrm{~min}$ and returned to control values at $90 \mathrm{~min}$. This can be explained by the appearance of barbiturate spikes (Fig. 3 , top panel) during this period. These barbiturate spikes are asymmetrical in their waveforms. Although barbiturate spikes gradually disappeared by $90 \mathrm{~min}$, the rat was still under heavy influence of the anesthetics at this point. This is best illustrated by the shaking and staggering movements of the rat. In a previous study (Shaw et al., 1999), we showed that pain-related cortical potentials returned to the control pattern only $180 \mathrm{~min}$ after administration of intraperitoneal pentobarbital. This correlates nicely with the recovery pattern of the complexity value (Fig. 3, lower panel).
There was a special transient period starting from the moment sodium pentobarbital was administered until 7 min later. During this period, outwardly, the rat became more excited and restless immediately after the anesthetic was given and gradually became quiet, finally losing consciousness. This biphasic change of the rat, i.e. a seemly increase followed by a decrease of its consciousness, was only tracked by the complexity index and not by the other four amplitude indices. For these four amplitude indices, only variance showed a significant change during this period. Even with the variance, the temporal pattern of the change was shifted to the right (2nd trace in Fig. 4, compare the positive peaks of variance versus complexity), and the change was monophasic.

To better illustrate the power of the complexity index in representing the state change during this transient period, the cortical activity of the data segment of one rat was sequentially shown minute-wise from top to bottom for a 7-min period after pentobarbital administration at the left portion of Fig. 5. The corresponding average complexity values of 4 rats are shown at the right side of Fig. 5. Immediately after pentobarbital injection (1st and 2nd min), EEG showed a desynchronous high-frequency pattern, shifted to a high-amplitude synchronization activity (biphasic, the $3 \mathrm{rd}-5$ th 
min), and then gradually became the sharp barbiturate spike pattern (the $6-7$ th $\mathrm{min}$ ). The average complexity values presented a significant trend for the dynamic changes of the brain functional states (one-way ANOVA, $P<0.001)$. In the time-frequency analysis, high complexity values corresponded to increased high-

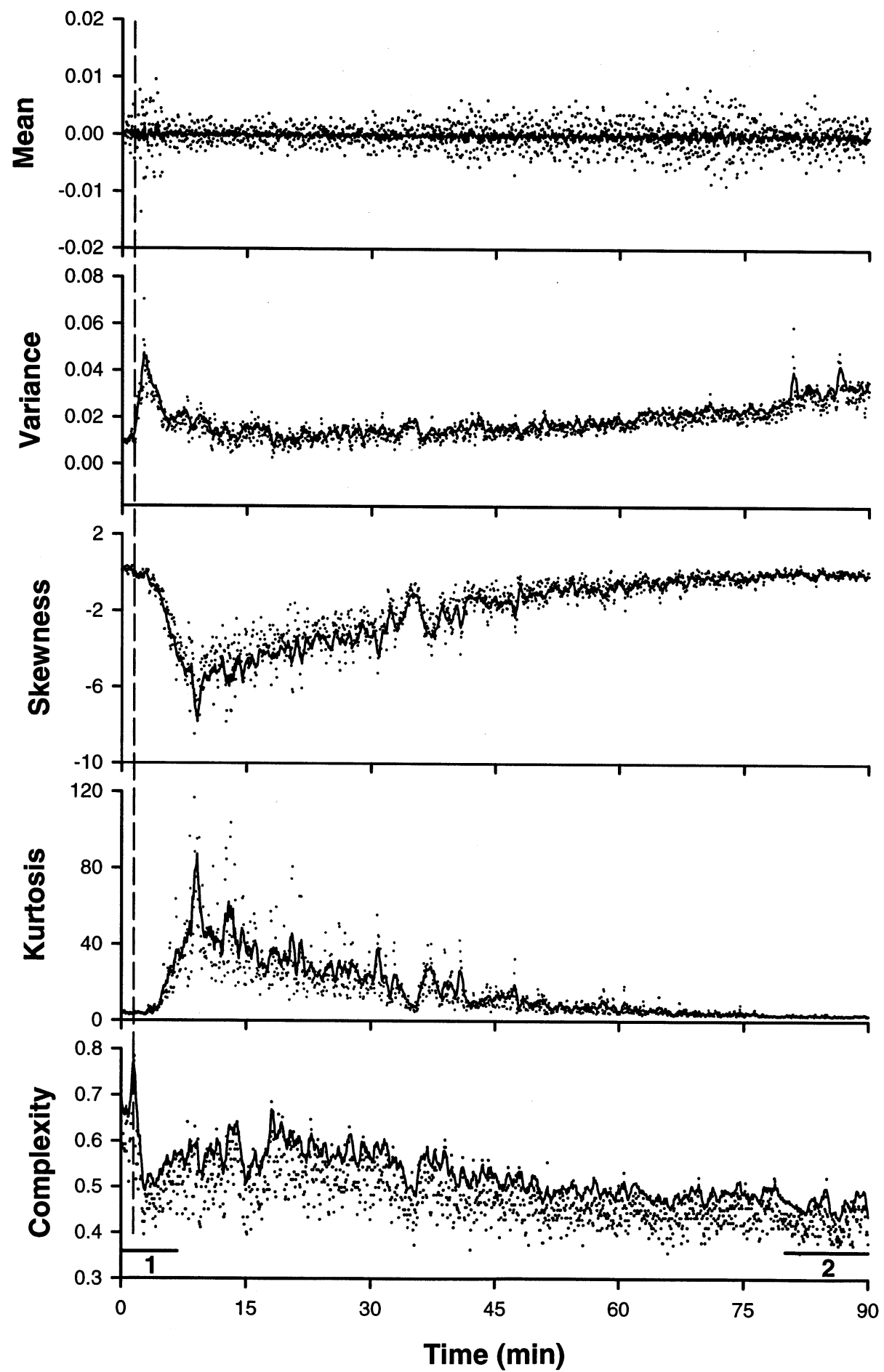

Fig. 4. Complexity values and four statistical moments of the awake-anesthetized EEG. Values (black dots) of complexity and four statistical moments were calculated from 2000 selected data segments with $50 \%$ overlap. Ten-point (solid line) moving averages were used to smooth the original trial. In a transient period from wakefulness to loss of consciousness, the complexity value shows a biphasic change (increasing initially then decreasing). There is a transient increase in the 2nd statistical moment (variance) analysis, but the response time is slower than in the complexity analysis (dashed line). The trends of skewness and kurtosis of the EEG (barbiturate spike dominant) in the range of 10-80 min after pentobarbital administration show significant differences to the awake and quasi-anesthetized states. Horizontal bars mark different behaviors: 1 , the stage from wakefulness to anesthesia; 2, movements of the head and tongue. 


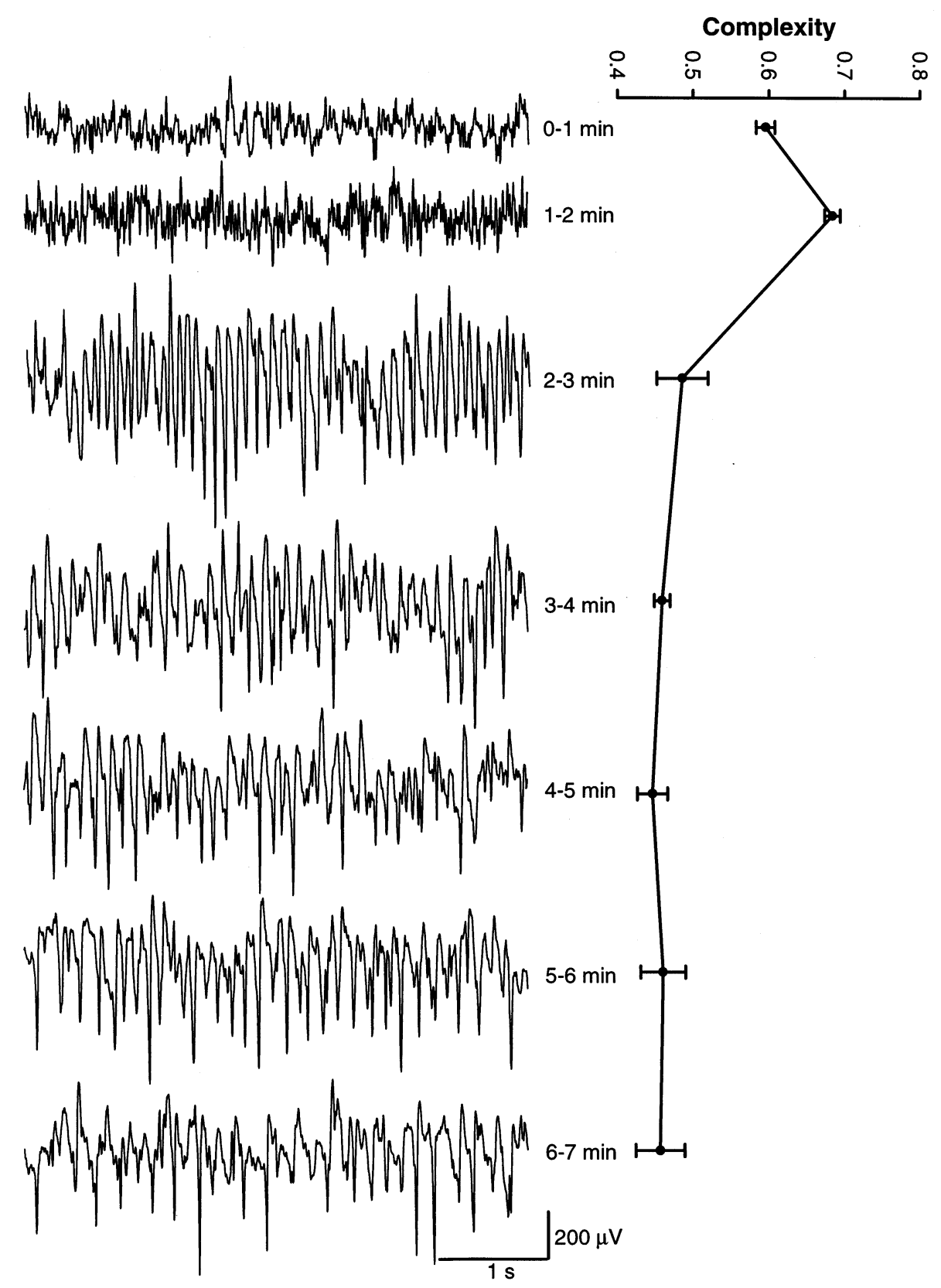

Fig. 5. Temporal activities and complexity value changes of the EEG in the first 7 min of the transition from wakefulness to anesthesia. Seven segments of EEG activities are sequentially illustrated after intraperitoneal pentobarbital administration from top to bottom. Note that synchronous activities were biphasic at first (3rd and 4th traces), changing to monophasic gradually (6th and 7th traces). The averaged complexity values $(n=4)$ are shown in the relative position of the temporal sequence on the right. The average complexity values presented a significant trend in this transient state (one-way ANOVA, $P<0.001$ ). Values are means $\pm \mathrm{SE}$.

frequency activity (indicated at 1 st -2 nd min of Fig. 6). The minimal complexity values at the $3 \mathrm{rd}-4$ th minutes corresponded to a transient period of strong low-frequency power with the biphasic EEG pattern. The slight increase in complexity values at the 6 th -7 th minutes corresponded to the unipolar high voltage spike period. Rats showed mild excitement in the induction phase during intraperitoneal pentobarbital administration (Wixson et al., 1987). This might be the reason why the high-frequency component of the cortical ac- tivity increased just before the animal lost consciousness. In another interpretation, we could image an external force (pentobarbital) acting on the central nervous system to interrupt/force the system into another functional state (anesthesia or loss of consciousness). The system showed transient bifurcation during this period. Therefore, high complexity of cortical potential was recorded.

There are several methods to calculate Komogorov's complexity of the symbol sequence. The algorithm used 


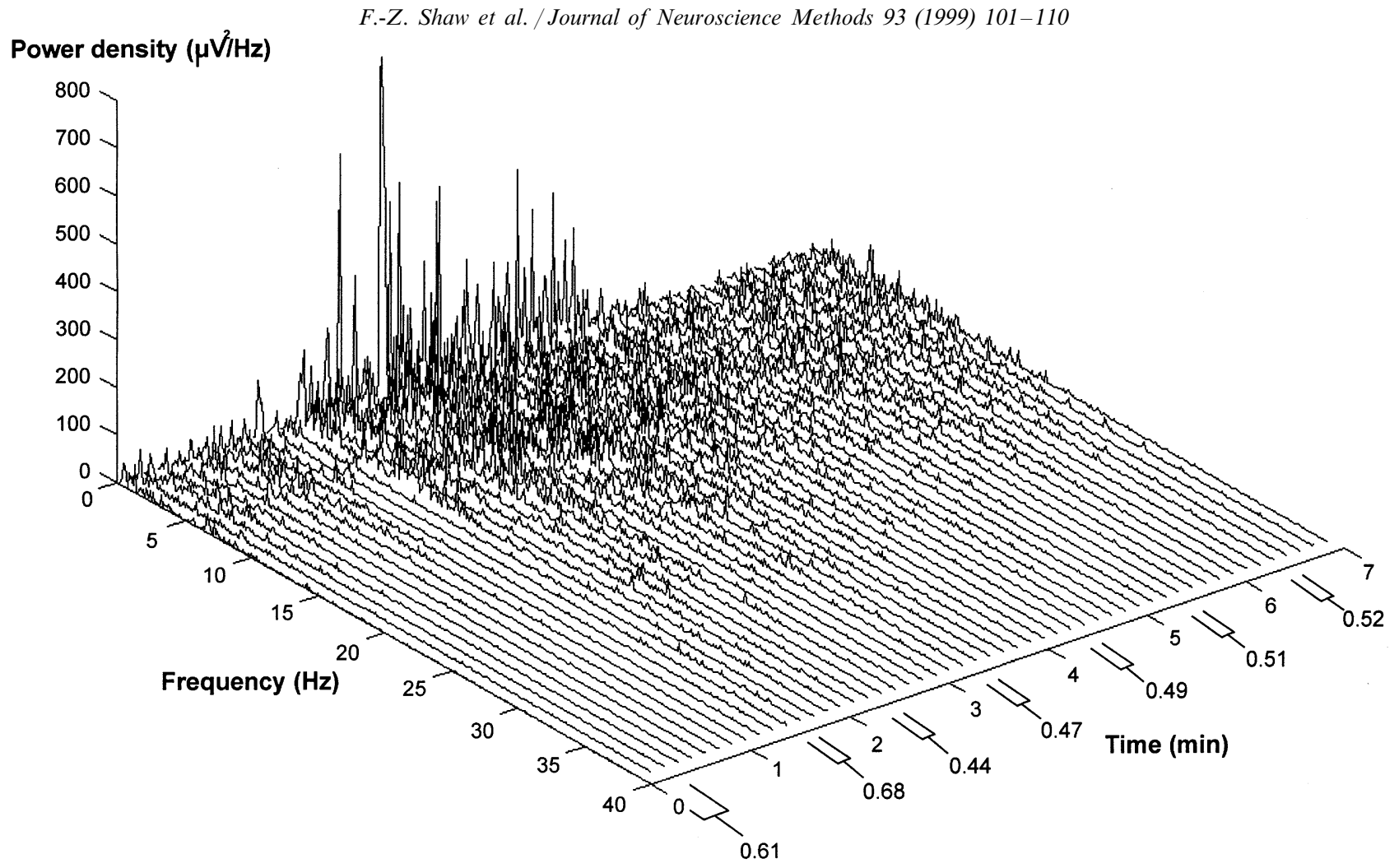

Fig. 6. Time-frequency characteristics and related complexity during the first $7 \mathrm{~min}$ of the wakefulness-to-anesthesia period. The spectral distribution shifted to a higher frequency (2nd $\mathrm{min}$ ) with the highest complexity value (numbers along the time axis), followed by high amplitude low frequency (3rd and 4th $\mathrm{min}$ ).

in this study, as proposed by Lempel and Ziv (1976), is composed of two operations (copy and insert). Lempel and Ziv (1976) demonstrated that this algorithm is an appropriate measure of Kolmogorov's complexity. Another algorithm, which uses repetitive 'copy' (Rapp et al., 1994), resulted in a similar characteristic for the randomly generated signal (compare Table 1 here with Table 1 of Rapp et al., 1994). Both show robust characteristics for the signal with/without phase randomized surrogates. Comparing the two methods, the algorithm here has the advantage of being easier to implement. Destexhe (1990) introduced a third algorithm, which is based on the Markov chain, to calculate the complexity of the potential spike interval of the pathological EEG. But spike waves are not common in EEG of normal condition. And we showed here that the complexity values of the amplitudes of the EEGs had significant differences in different functional states (Fig. 2). Therefore, we used amplitude fluctuation rather than spike interval to calculate the algorithmic complexity.

We have described the algorithmic complexity as a dynamic estimator of cortical functions in this investigation. It needs only simple calculations, and its value is relatively data-length independent. Different functional states were tested and significant differences were found in their complexity values. Continuous tracking of the functional changes of the brain before and after pentobarbital administration in chronically implanted animal shows intricate differences during the induction phase using complexity values. These differences correlated with behavioral observations and EEG spectral characteristics. Therefore, we conclude that this simple dynamic index can be used to quantitatively analyze the cortical functions of rats.

\section{Acknowledgements}

We thank Daniel P. Chamberlin for English editing. The present study was supported by grants NSC852311-B002-031 and NSC88-2311-B002-038 from the National Science Council of the Republic of China.

\section{References}

Basar E. Chaos in brain function. Berlin Heidelberg, New York: Springer-Verlag, 1990

Chaitin GJ. Information theoretical computational complexity. IEEE Trans Inform Theory 1974;20:10-5.

Destexhe A. Symbolic dynamics from biological time series. Phys Lett A $1990 ; 143: 373-8$.

Eckmann JP, Ruelle D. Fundamental limitations for estimating dimensions and liapunov exponents in dynamic systems. Physica D 1992;56:185-7. 
Elbert T, Ray WJ, Kowalik ZJ, Skinner JE, Graf KE, Birbaumer N. Chaos and physiology: deterministic chaos in excitable cell assemblies. Physiol Rev 1994;74:1-47.

Grassberger P, Procaccia I. Characterization of strange attractors. Phys Rev Lett 1983;50:346-9.

Kaspar F, Schuster HG. Easily calculable measure for the complexity of spatiotemporal patterns. Phys Rev A 1987;36:842-8.

Kolmogorov AN. Three approaches to the definition of the concept of quantity of information. IEEE Trans Inform Theory 1965; 14:662-9.

Lempel A, Ziv J. On the complexity of finite sequences. IEEE Trans Inform Theory 1976;22:75-81.

Paulus MP, Geyer MA, Gold LH, Mandell AJ. Application of entropy measure derived from the ergodic theory of dynamic systems to rat locomotor behavior. Proc Natl Acad Sci USA 1990;87:723-7.

Rapp PE, Zimmerman ID, Vining EP, Cohen N, Albano AM, Jimenez-Montano MA. The algorithmic complexity of neural spike trains increases during focal seizures. $\mathbf{J}$ Neurosci 1994;14:4731-9.
Roschke J, Fell J, Beckmann P. The calculation of the first positive Lyapunov exponent in sleep EEG data. Electroenceph Clin Neurophysiol 1993;86:348-52.

Shaw FZ, Chen RF, Tsao HW, Yen CT. A multichannel system for recording and analysis of cortical field potentials in freely moving rats. J Neurosci Methods 1999;88:33-43.

Steriade M, Jones EG, Llinas RR. Thalamic Oscillations and Signaling. New York: Wiley, 1990.

Theiler J, Rapp PE. Re-examination of the evidence for low-dimensional, non-linear structure in the human electroencephalogram. Electroencephalogr Clin Neurophysiol 1996;98:213-22.

Wixson SK, White WJ, Hughes HC Jr, Lang CM, Marshall WK. A comparison of pentobarbital, fentanyl-droperidol, ketamine-xylazine and ketamine-diazepam anesthesia in adult male rats. Lab Anim Sci 1987;37:726-30.

$\mathrm{Xu} \mathrm{JH}, \mathrm{Wu} \mathrm{XB}$. Using complexity measure to characterize information transmission of human brain cortex. Sci China B Life Sci Earth Sci 1994;37:1455-62. 